\title{
WILEY-VCH
}

Exciton and Charge Carrier Dynamics in Highly Crystalline PTQ10:IDIC Organic Solar Cells.

Hyojung Cha*, Yizhen Zheng, Yifan Dong, Hyun Hwi Lee, Jiaying Wu, Helen Bristow, Jiangbin Zhang, Harrison Ka Hin Lee, Wing C. Tsoi, Artem A. Bakulin, Iain McCulloch, James R. Durrant*

Dr. H. Cha, Y. Zheng, Y. Dong, Dr. J. Wu, H. Bristow, J. Zhang, Dr. A. A. Bakulin, Prof. I. McCulloch, Prof. J. R. Durrant Department of Chemistry and Centre for Plastic Electronics, Imperial College London, London W12 0BZ, United Kingdom.

E-mail: h.cha@imperial.ac.uk, j.durrant@imperial.ac.uk

Dr. H. H. Lee

Pohang Accelerator Laboratory (PAL), Pohang University of Science and Technology (POSTECH), Pohang, Gyeongbuk 37673, Republic of Korea.

\section{J. Zhang}

Cavendish Laboratory, University of Cambridge, JJ Thomson Avenue, Cambridge CB3 OHE, United Kingdom

Dr. H. K. H. Lee, Dr. W. C. Tsoi, Prof. J. R. Durrant

SPECIFIC IKC, College of Engineering, Swansea University, Bay Campus, Fabian Way, Swansea, Wales, SA1 8EN, United Kingdom.

Prof. I. McCulloch

King Abdullah University of Science and Technology (KAUST), KAUST Solar Center (KSC), Thuwal, 23955-6900, Saudi Arabia.

Keywords: organic solar cells; nonfullerene acceptor; charge separation; recombination; phase separation

\begin{abstract}
Herein we investigate the morphology and exciton / charge carrier dynamics in bulk heterojunctions (BHJs) of the donor polymer PTQ10 and small molecule acceptor IDIC. PTQ10:IDIC BHJs have been shown to be particularly promising for low cost organic solar cells (OSCs). We find that both PTQ10 and IDIC show remarkably high crystallinity in optimised BHJs, with GIWAXS data indicating $\pi-\pi$ stacking coherence lengths of up to $8 \mathrm{~nm}$. Ultrafast exciton-exciton annihilation studies indicate long exciton diffusion lengths for both
\end{abstract}




\section{WILEY-VCH}

neat materials (19 nm for PTQ10 and $9.5 \mathrm{~nm}$ for IDIC). These long exciton diffusion lengths enable efficient exciton separation, as measured by photoluminescence quenching experiments, despite the high degree of phase segregation in this blend. Photoinduced charge generation from PTQ10 and IDIC excitons is observed, with half times of 1 and 3 ps, respectively, in both cases most likely determined by the kinetics of exciton diffusion to domain interfaces. Transient absorption data indicates that exciton separation leads to the formation of two spectrally distinct species, assigned to interfacial charge transfer (CT) states and separated charges. CT state decay is correlated with the appearance of additional separate charges, indicating relatively efficient CT state dissociation, attributed to the high crystallinity of this blend. Our results emphasis the potential for high material crystallinity to enhance charge separation and collection in OSCs, but also that long exciton diffusion lengths are likely to be essential for efficient exciton separation in such high crystallinity devices. 


\section{WILEY-VCH}

\section{Introduction}

Organic solar cells (OSCs) based on donor:acceptor bulk heterojunctions (BHJs) are a promising photovoltaic technology due to their potential for low cost, light weight, flexible and integratable devices. ${ }^{[1-4]}$ In such BHJs, typically comprising a blend of an electron donating conjugated polymer and an electron accepting small molecule, pure phases of donor and/or acceptor often coexist alongside a molecularly intermixed donor:acceptor phase. ${ }^{[5-9]}$ The overall blend morphology, and the proportions and lengthscales of such pure and intermixed domains have been shown to be critical in determining device performance. ${ }^{[5-9]}$ In recent years, the development of a range of nonfullerene acceptors (NFAs) has enabled, when blended with complementary absorbing donor polymers, impressive advances in OSC performance. ${ }^{[10,11]}$ In the study herein, we focus on the recently reported PTQ10:IDIC blend system, which has been suggested to be particularly promising for low cost commercial application. ${ }^{[2]}$ We find this blend system to be remarkably crystalline, and investigate the impact of this high crystallinity upon the exciton and charge carrier dynamics determining device performance.

In BHJ blends, a large donor/acceptor interfacial area facilitates the exciton dissociation required for efficient photocurrent generation. ${ }^{[12,13]}$ However, this donor/acceptor interface can also yield interfacial electron-hole pairs, often referred to as charge transfer (CT) states. ${ }^{[12,14-17]}$ CT states formed in the highly intermixed regions of such blends have been reported to give rise to coulombically bound $\mathrm{CT}$ states, potentially resulting in geminate $\mathrm{CT}$ state recombination. In contrast, it has been reported that CT states formed at the interface between crystalline, pure domains tend to give rise unbound $\mathrm{CT}$ states which are more readily for efficient charge generation. ${ }^{[12,18-24]}$ The proportion of intermixed and pure domains depends strongly upon material crystallinity ${ }^{[25,26]}$, film processing ${ }^{[27]}$ and blend ratio $^{[28]}$. In polymer:fullerene blends, increasing the fullerene content has been shown to result in a transition from a molecularly intermixed polymer:fullerene single phase to the coexistence of mixed and pure PCBM phases. ${ }^{[21,29,30]}$ For example, PCDTBT:PCBM blends show an ultrafast exciton separation 


\section{WILEY-VCH}

process populating CT states in the intermixed phase, with the dissociation of these CT states into free charges only being observed in the presence of pure fullerene domains. ${ }^{[20]}$ This improvement in electron-hole separation yield has been assigned to the generation of more spatially delocalized CT states at the interfaces with pure fullerene domains, ${ }^{[31-33]}$ as well as an energetic shift of fullerene LUMO level between intermixed and pure domains. ${ }^{[21,22]}$ In addition, a correlation between interfacial face-on molecular orientation and CT energetics has been recently observed in small molecule / (C60) bilayer devices. ${ }^{[45]}$ Whilst the dependence of charge generation and device performance on the presence of pure acceptor domains is now well established for fullerene acceptors such as $\mathrm{PCBM},{ }^{[6,34]}$ the importance of such domains is less well established for devices employing the increasingly studied NFA systems. A face-on molecular orientation of the electron accepting polymer N2200 has been reported to increase energy losses and decrease open circuit voltage in all polymer bilayer devices, ${ }^{[4]}$ in contrast to that observed for small molecule NFA's. ${ }^{[48]}$ For small molecule NFAs attention has focused particularly on controlling energy offsets to optimize performance. ${ }^{[48]}$ A strong NFA $\pi-\pi$ stacking has been reported to enhance charge transport, but not linked systematically with charge generation. ${ }^{[48]}$ Therefore ultrafast spectroscopic studies have shown that charge carriers are also generated efficiently in NFA-based $\mathrm{OSCs}^{[35-40]}$, but a detailed photophysical understanding of NFA OSCs still lags behind the continuing, very promising, advances in their device efficiencies.

NFAs are attracting notable attention due to their advantages of easy synthesis and purification, strong and broad light absorption, tuneable bandgap and energy levels, and better device stability compared to fullerene electron acceptors, yielding power conversion efficiencies (PCEs) of over $16 \%$ for single junction OSCs. ${ }^{[2,41,42]}$ Several morphological studies have indicated that the presence of small pure phases of NFA in polymer:NFA blends can have a beneficial impact on device performance, attributed to minimised recombination losses. ${ }^{[24,25,28,43]}$ In addition, it has been reported that an energetic cascade between mixed and 


\section{WILEY-VCH}

pure phases in a relatively intermixed polymer:NFA blend can drive photogenerated electrons to the pure phase, thereby facilitating the spatial separation of charges and reducing CT state recombination losses. ${ }^{[28]}$ Most previous transient kinetic studies have addressed charge carrier dynamics in polymer:NFA blends employing relatively low crystallinity NFAs such as FBR, ${ }^{[44]}$ IDFBR, $^{[38]}$ and IDTBR $^{[28]}$, reporting tightly bound CT states in the intermixed blend morphology, with the dissociation of these states being enhanced by the presence of aggregated NFA domains. Such behaviour is broadly analogous to that observed for polymer:PCBM blends. ${ }^{[9,22,29,45]}$ However, promising device performance has also recently been reported for increasingly crystalline NFAs which exhibit increased molecular self-assembly features with a preference of face-on molecular packing orientation. ${ }^{[46-48]}$ It has been suggested this orientation will be beneficial for efficient charge transport ${ }^{[49]}$, however few studies have addressed the impact of molecular orientation on charge separation. Herein we address how interfacial morphology and NFA crystallinity impact upon charge generation and device performance in a high crystallinity conjugated polymer:NFA blend.

This study focuses on recently reported low-cost and high performance OSCs based on a blend of donor polymer PTQ10 and NFA IDIC (see Figure 1a for molecular structures and full names in supporting information), yielding PCEs of $12.7 \%$ along with a high open circuit voltage $\left(V_{\mathrm{OC}}\right)$ of $0.97 \mathrm{~V}$, with the latter being attributed to the deep HOMO level of PTQ10. ${ }^{[2,42]}$ PTQ10 is of particular interest due to its ease of synthesis, with a high overall product yield of $87.4 \%$ using only two synthetic steps from a commercially available intermediate. In addition, morphological studies have indicated that PTQ10:IDIC blends exhibit face-on orientated and close $\pi-\pi$ stacking, and a high crystallinity after thermal annealing and/or solvent vapour treatment. ${ }^{[2,42]}$ However, studies of underlying exciton and charge carrier dynamics in the photoactive layers employing PTQ10:IDIC blend, and particularly how these charge carrier dynamics are influenced by blend morphology, have been absent from the literature to date. In this study herein, we have undertaken a study of the morphology of PTQ10:IDIC blends and its 
impact upon the underlying charge separation and recombination dynamics and resulting in solar cell performance.

(a)

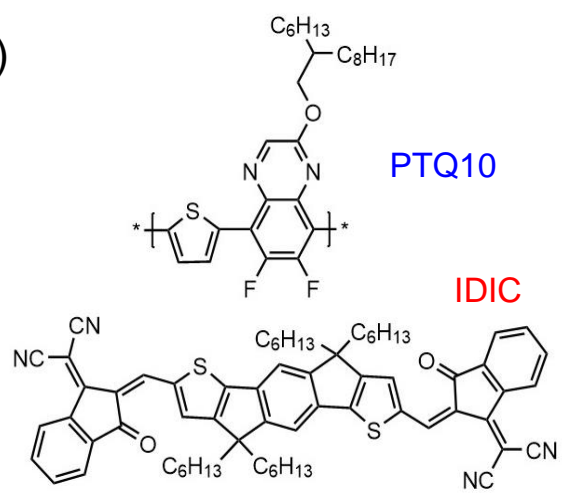

(c)

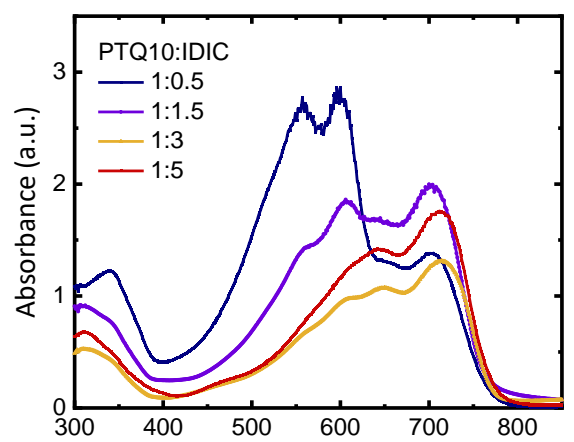

(e)

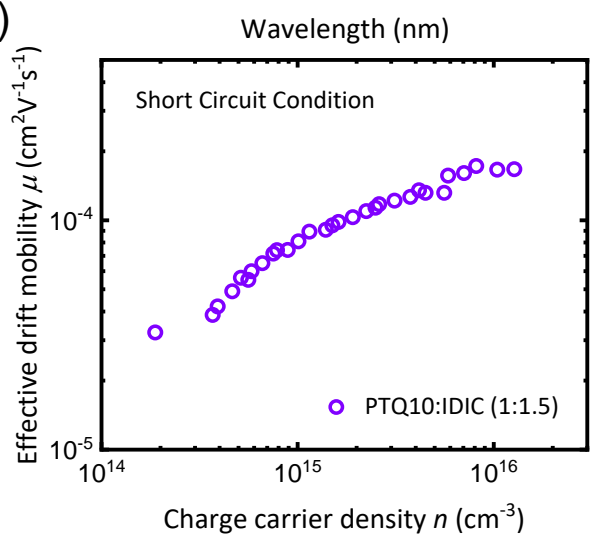

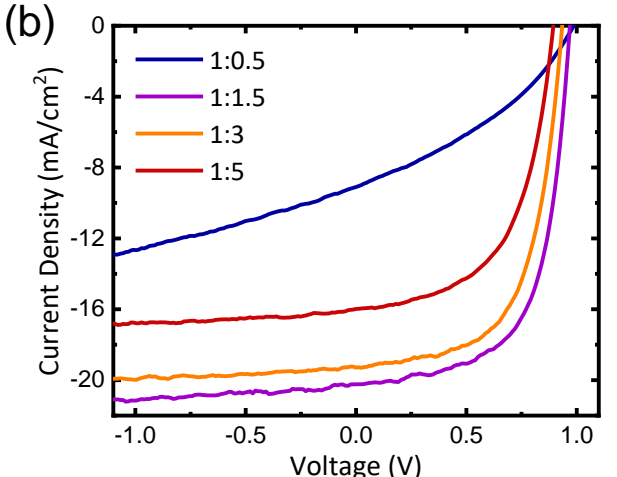

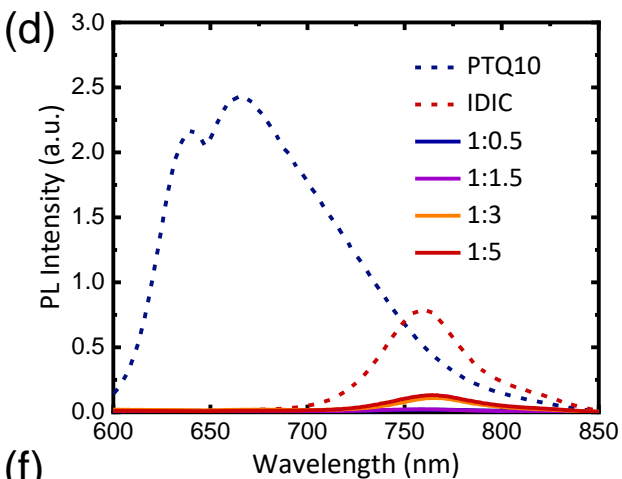

(f)

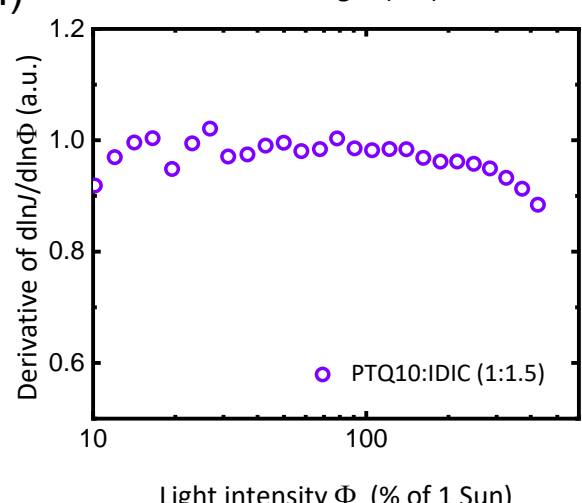

Figure 1. (a) Molecular structure of PTQ10 and IDIC. (b) The $J-V$ curve of PTQ10:IDIC blend solar cells with various composition ratios under illumination of $A M 1.5 \mathrm{G}, 100 \mathrm{~mW} \mathrm{~cm}{ }^{-2}$. (c) UV-visible absorbance spectra for PTQ10:IDIC blend films with various composition ratios. (d) PL spectra excited at $450 \mathrm{~nm}$ for neat PTQ10 and IDIC films and PTQ10:IDIC blend films with various composition ratios. (e) Effective mobility for optimised composition determined from charge extraction measurements at short circuit (1:1.5). (f) Linearity analysis of short circuit current as a function of light intensity of the optimised 1:1.5 PTQ10:IDIC device. 


\section{WILEY-VCH}

\section{Results}

\subsection{Photovoltaic Performance and Steady State Optical Properties}

Solution-processed organic photovoltaic devices were fabricated using an inverted device structure of ITO/ZnO/PTQ10:IDIC/ $\mathrm{MoO}_{3} / \mathrm{Ag}$. Photoactive layers were spin-coated with PTQ10:IDIC blend solutions (total concentration of $30 \mathrm{mg} \mathrm{ml}^{-1}$ dissolved in chloroform) with blend ratios ranging from 1:0.5 to 1:5 to modulate blend morphology. Current density-voltage $(J-V)$ characteristics of PTQ10:IDIC devices are shown in Figure 1b and summarised in Table S1. PTQ10:IDIC blend devices with a ratio of 1:1.5 exhibited optimal photovoltaic performance; open circuit voltage $\left(V_{\mathrm{OC}}\right)$ of $0.97 \mathrm{~V}$, current density $\left(J_{\mathrm{SC}}\right)$ of $20.2 \mathrm{~mA} \mathrm{~cm}{ }^{-2}$, fill factor $(\mathrm{FF})$ of $63 \%$, and PCE of $12.4 \%$, in agreement with literature data. We note that higher efficiency PTQ10:IDIC devices have been reported using a conventional (p-i-n) device structure. ${ }^{[2]}$ The study herein focuses on the inverted n-i-p structure, the structure typically employed in OSCs with NFAs due to the enhanced device stability. It is apparent from Figure $1 \mathrm{~b}$ that as the concentration of IDIC increases, $V_{\mathrm{OC}}$ decreases. Following previous reports of other material systems, ${ }^{[9,18,28]}$ this $V_{\mathrm{OC}}$ loss could result from an increase in LUMO of IDIC caused by IDIC aggregation/crystallisation. Such a shift of LUMO energy of IDIC is consistent with red-shifted IDIC emission peaks in blends with high IDIC ratio reported in photoluminescence (PL) and electroluminescence (EL) spectra $^{[20,50]}$, as well as red-shifted absorption onset wavelengths, as shown in Figure 1c, S1 and S2.

Absorption spectra of neat PTQ10 and IDIC films along with their PL spectra are shown in Figures 1 and S2. PTQ10 mainly absorbs light with wavelength up to $600 \mathrm{~nm}$, whilst IDIC shows broad absorption from $550 \mathrm{~nm}$ to $750 \mathrm{~nm}$. Their complementary absorption features enable PTQ10:IDIC blends to exhibit spectrally broad light absorption, enabling efficient harvesting of solar irradiation (Figure 1c). The blends show high external quantum efficiencies (EQE) across nearly entire visible region (Figure S3), in agreement with their absorption profiles, with EQEs of up to $75 \%$ for the 1:1.5 blend. Figure 1d displays PL spectra for neat 


\section{WILEY-VCH}

material and blend films corrected by their absorbance at the $450 \mathrm{~nm}$ excitation wavelength. Efficient photoluminescence quenching (PLQ) is observed in all blends, indicating efficient exciton dissociation via electron and / or hole transfer, as quantified in Table S2. Electron transfer from PTQ10 to IDIC in all the four composition films is highly efficient, showing over 99\% PL quenching (we note this quenching may also result in part from energy transfer followed by hole transfer), whilst hole transfer process from IDIC to PTQ10 is less efficient when the ratio of PTQ10:IDIC is 1:3 and 1:5 in a blend (87 and 84\% PL quenching respectively), indicating that excitons generated in IDIC domains find it more difficult to reach to the donor/acceptor interface, indicative of the presence of larger pure domains of IDIC at high IDIC blend compositions. Device PL and EL spectra (Figure S2) are dominated by IDIC exciton emission, with the exception of the 1:0.5 blend which exhibits red shifted emission indicative of the radiative recombination of interfacial CT states.

The charge carrier transport and collection properties were investigated in the optimised PTQ10:IDIC (1:1.5) device. The effective drift mobility ( $\mu$ ) of a PTQ10:IDIC (1:1.5) solar cell was calculated from measurements of charge carrier density at short-circuit $\left(n_{\mathrm{SC}}\right)$ measured by charge extraction technique (CE). Most NFA-based solar cells show relative low effective mobilities on the order of $10^{-5} \mathrm{~cm}^{2} \mathrm{~V}^{-1} \mathrm{~s}^{-1}$ measured by the same technique, which is typically assigned to lack of continuous phases for charge transport. ${ }^{[51-53]}$ In contrast, this PTQ10:IDIC blend shows a high effective mobility as $2 \times 10^{-4} \mathrm{~cm}^{2} \mathrm{~V}^{-1} \mathrm{~s}^{-1}$ (Figure 1e), higher than other NFAs blends ${ }^{[2,54-56]}$, and consistent with previous reports $\left(1.1 \times 10^{-3} \mathrm{~cm}^{2} \mathrm{~V}^{-1} \mathrm{~s}^{-1}\right.$ extracted from space charge limited current measurements from electron-only devices for IDIC which is close to those $\left(10^{-3} \mathrm{~cm}^{2} \mathrm{~V}^{-1} \mathrm{~s}^{-1}\right)$ of fullerene acceptors $\left.{ }^{[57]}\right)$. This high mobility is consistent with the reported face-on orientation of crystallites in this blend, favourable for efficient charge transport

and collection. ${ }^{[58,59]}$ Supporting this conclusion, analysis of the linearity of short-circuit photocurrent as a function of light intensity (plotted in derivative form in Figure 1f) shows efficient photocurrent collection up to 2 Sun illumination intensity without linearity losses. This 


\section{WILEY-VCH}

indicates negligible non-geminate recombination losses during collection at short circuit up to this light intensity, also consistent with a nanomorphology favouring efficient charge transport and collection.
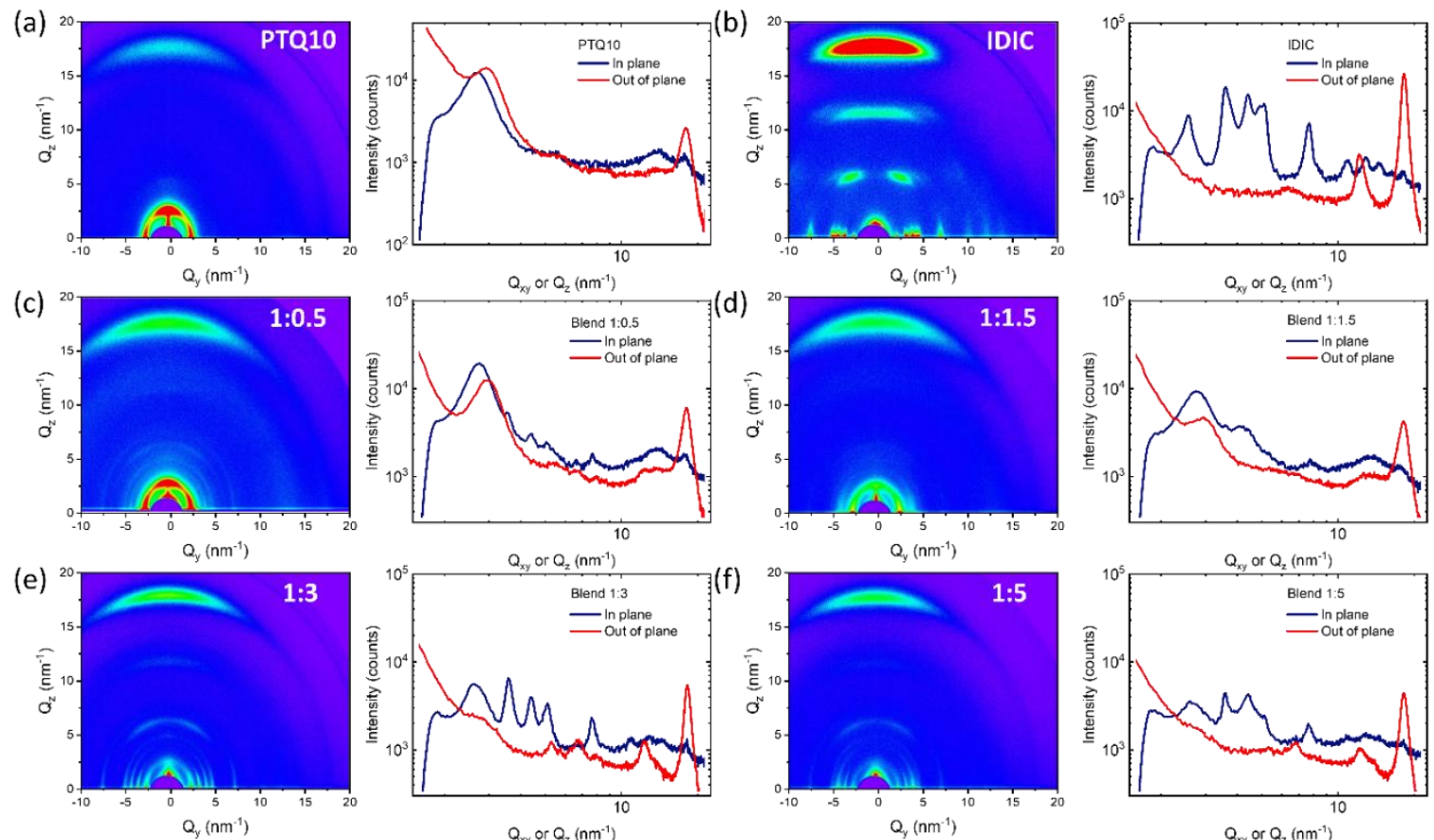

Figure 2. GIWAXS images (left) and line-cuts (right) of (a) neat PTQ10 film, (b) neat IDIC film, and PTQ10:IDIC blend films of (c) 1:0.5, (d) 1:1.5, (e) 1:3, and (f) 1:5 ratios, respectively.

\subsection{Photoactive Materials Morphology}

We next investigate the molecular orientation/phase separation features in these PTQ10:IDIC blends and how these correlate with device performance. We probed the microstructural differences between PTQ10:IDIC blend layers with blend ratios ranging from 1:0.5 to 1:5 using grazing incidence wide angle X-ray scattering (GIWAXS). The GIWAXS images and line-cut plots of neat PTQ10 and IDIC films and PTQ10:IDIC blend films are presented in Figure 2. As shown in Figure 2a,b, for the neat PTQ10 and IDIC films, (010) peaks were observed at $18.0 \mathrm{~nm}^{-1}$ and $18.21 \mathrm{~nm}^{-1}$ in out-of-plane direction, which correspond to $\pi-\pi$ stacking distances of $3.49 \AA$ and $3.45 \AA$ respectively. Both PTQ10 and IDIC films show a preferential face-on orientation on the substrate. ${ }^{[2]}$ The neat IDIC film displays sharper diffraction peaks in the inplane direction and stronger and sharper $\pi-\pi$ stacking peaks in out-of-plane direction in 


\section{WILEY-VCH}

comparison with PTQ10. In addition, IDIC exhibits strong lamellar structure with the (001) peak in the in plane direction, corresponding to dominant stacking of IDIC molecules (spacing $\sim 24.5 \AA$ ) in the neat film. All the PTQ10:IDIC blends also showed well-defined $\pi$ - $\pi$ stacking with (010) diffraction peaks in out-of-plane direction, demonstrating in these blends a preferential face-on orientation of both PTQ10 and IDIC crystallites relative to the substrate. The $\pi-\pi$ stacking distance in PTQ10:IDIC film of 1:0.5 ratio was $3.48 \AA$ calculated at the $(010)$ peak of $18.06 \mathrm{~nm}^{-1}$ which is closer to the $\pi-\pi$ stacking distance of neat PTQ10 film (Figure 2c and Table S3). As the concentration of IDIC increases, the (010) peaks are gradually shifted to $18.18 \sim 18.23 \mathrm{~nm}^{-1}$ corresponding to dominant $\pi-\pi$ stacking of IDIC $(\sim 3.45 \AA)$ in the blend films (Figure 2d-f). Interestingly the PTQ10:IDIC film with 1:1.5 ratio, which showed optimised device performance, exhibits both the (100) peak corresponding to PTQ10 lamellar packing structure and the (010) peak corresponding to $\pi-\pi$ stacking of IDIC, which confirms that both PTQ10 and IDIC form molecularly ordered structures at this blend composition. Moreover, these (010) diffraction peaks enable us to extract quantitatively the $\pi-\pi$ stacking coherence lengths of the PTQ10 and IDIC domains using the Scherrer equation. ${ }^{[60]}$ This analysis yields a $\pi-\pi$ stacking coherence length of PTQ10 domains in neat PTQ10 of $8.5 \mathrm{~nm}$ and in the 1:0.5 blend of $5.0 \mathrm{~nm}$ (this length could not be clearly determined for the lower PTQ10 composition blends). The $\pi$ - $\pi$ stacking coherence length of IDIC was measurable for all blend films, increasing from $8.2 \mathrm{~nm}$ at $1: 1.5$ ratio to $10.7 \mathrm{~nm}$ at $1: 5$ ratio, exceptionally large values compared to other polymer:NFA blends ${ }^{[46,61]}$, and also larger than that observed for neat IDIC (7.2 nm). It has previously been reported that blends with larger (010) coherence lengths exhibit higher domain purity, and can yield higher device $J_{\mathrm{SC}}$ and FF. ${ }^{[46,61]}$ In addition, it is also noted that the PTQ10:IDIC system exhibits the longest $\pi-\pi$ stacking coherence lengths reported to date for polymer:NFA blends (e.g. ITIC-Th $=3.8 \mathrm{~nm}$ in PTB7-Th:ITIC-Th blend, and IT-M = $5.66 \mathrm{~nm}$ in PBDB-T:IT-M blend). ${ }^{[46,62,63]}$ For the PTQ10:IDIC blends studied herein, our data therefore indicate remarkably long distance $\pi-\pi$ stacking of IDIC molecules in the blend, 


\section{WILEY-VCH}

orientated parallel to the substrate and so well aligned to favour efficient charge transport. We note, as we show later, that the increased pure IDIC domain size limits the exciton dissociation yield, leading to lower $J_{\mathrm{SC}}$.
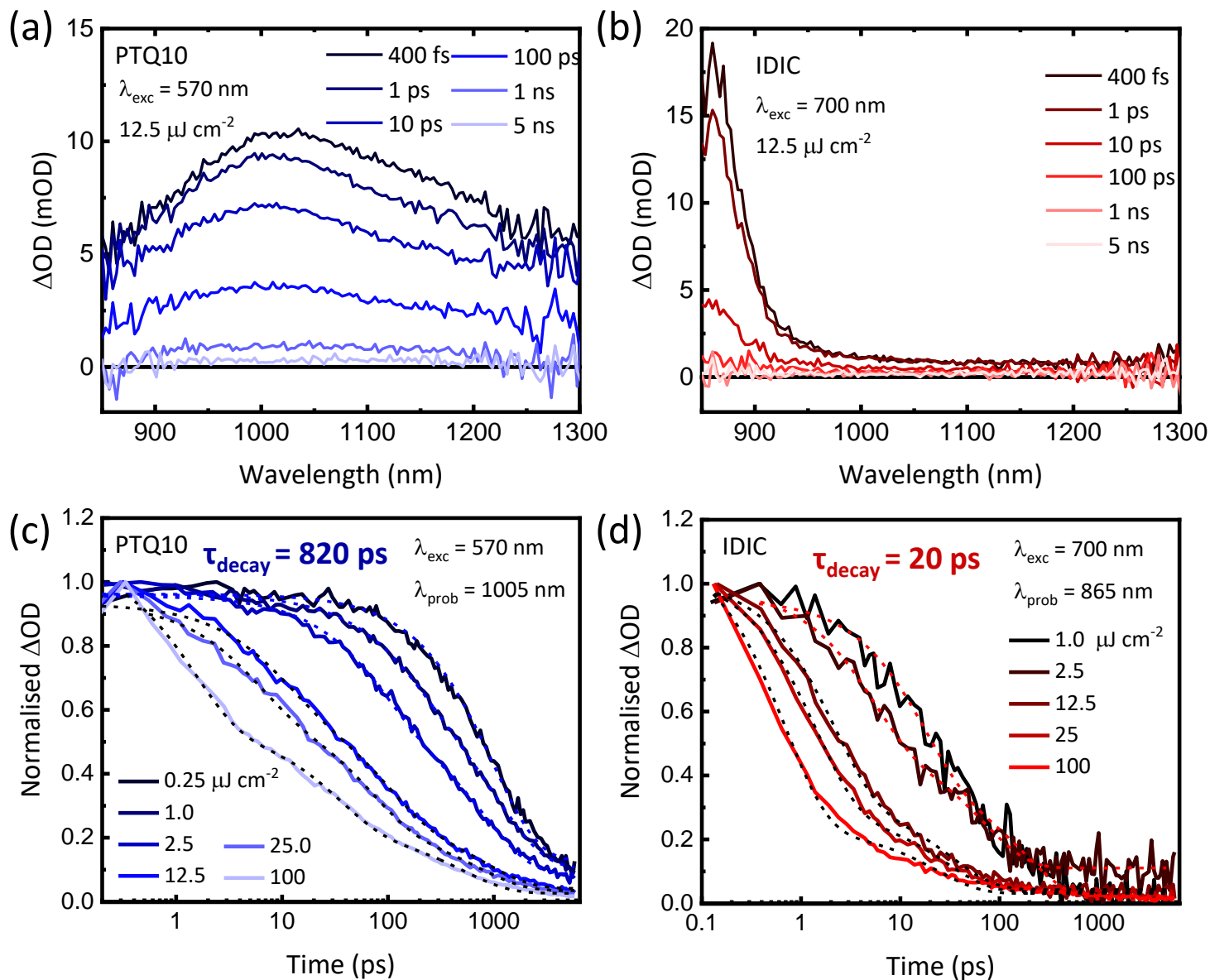

Figure 3. Transient absorption spectra for neat (a) PTQ10 and (b) IDIC films. PTQ10 is excited at 570 $\mathrm{nm}$ with energy density of $12.5 \mu \mathrm{J} \mathrm{cm}^{-2}$, and IDIC is excited at $700 \mathrm{~nm}$ with the identical energy density. Transient absorption decay kinetics for (c) the neat PTQ10 and (d) IDIC films under different carrier densities, probed at $1005 \mathrm{~nm}$ and $865 \mathrm{~nm}$, respectively.

\subsection{Transient Absorption Spectroscopy}

The strong $\pi-\pi$ stacking of PTQ10 and IDIC films might be expected to enhance exciton diffusion. In order to address these exciton dynamics, we employed intensity dependent ultrafast transient absorption spectroscopy (TAS) to measure the exciton lifetimes and calculate exciton diffusion lengths in neat PTQ10 and IDIC films. The method to determine the exciton diffusion coefficient $(D)$, monomolecular exciton lifetime $(\tau)$ and exciton diffusion length $\left(L_{\mathrm{D}}\right)$ 


\section{WILEY-VCH}

is based on the observation of the monomolecular exciton decay to the ground state at low excitation densities and bimolecular singlet exciton-singlet exciton annihilation at high excitation densities. ${ }^{[33,44]}$ Figure 3a,b exhibits ultrafast transient absorption spectra for neat PTQ10 and IDIC films. A broad exciton photoinduced absorption (PIA) peak of PTQ10 is observed centred at $1005 \mathrm{~nm}$, whilst that of the IDIC exciton is observed at around $865 \mathrm{~nm}$. The decay of these PIA features was observed to be strongly intensity dependent. For PTQ10, decay half-times range from 820 ps at the lowest excitation density of $0.25 \mu \mathrm{J} \mathrm{cm}^{-2}$ to 5.5 ps at $100 \mu \mathrm{J}$ $\mathrm{cm}^{-2}$, as shown in Figure 3c,d and Table S4. These decay dynamics are monoexponential at low excitation densities, whilst power laws are observed at high excitation densities. In addition, the longest exciton lifetime (820 ps) of PTQ10 is consistent with that measured by timecorrelated single-photon counting (TCSPC), as shown in Figure S4. From transient absorption data, as presented in table S4, we obtain values of $D=4.5 \times 10^{-3} \mathrm{~cm}^{2} \mathrm{~s}^{-1}$ and $L_{\mathrm{d}}=19.3 \mathrm{~nm}$ for PTQ10 film. This exciton diffusion length of $19 \mathrm{~nm}$ for PTQ10 is remarkably large for organic conjugated polymers, with most materials exhibit values in the range $5-10 \mathrm{~nm} \cdot{ }^{[5]}$ Our analysis indicates that this long exciton diffusion length results primarily from a long exciton lifetime for PTQ10 rather than fast exciton diffusion kinetics. The long exciton lifetime and exciton diffusion length for PTQ10 are likely to be the key reason behind blends employing this polymer being able to achieve efficient polymer PLQ, and therefore exciton separation, for a broad range of blend compositions (see Figure 1d above). We observed similar and high PTQ10 PL quenching for all blend compositions studied, indicating the efficiency of charge generation from PTQ10 excitons is not a limiting factor in these devices. Analogous data for neat IDIC yielded $L_{\mathrm{d}}=9.5 \mathrm{~nm}$, resulting from a high exciton diffusion coefficient $\left(D=4.4 \times 10^{-2} \mathrm{~cm}^{2} \mathrm{~s}^{-}\right.$ ${ }^{1}$ ), whilst IDIC showed a rather short exciton lifetime of $20 \mathrm{ps.} \mathrm{This} \mathrm{high} \mathrm{IDIC} \mathrm{exciton} \mathrm{diffusion}$ coefficient could result from its rigid planar molecular structure, strong $\pi-\pi$ stacking and high film crystallinity. ${ }^{[64-66]}$ The short exciton lifetime of IDIC most likely results, following the energy gap law, from its low optical bandgap, as well as potentially from its high 
crystallinity. ${ }^{[67]}$
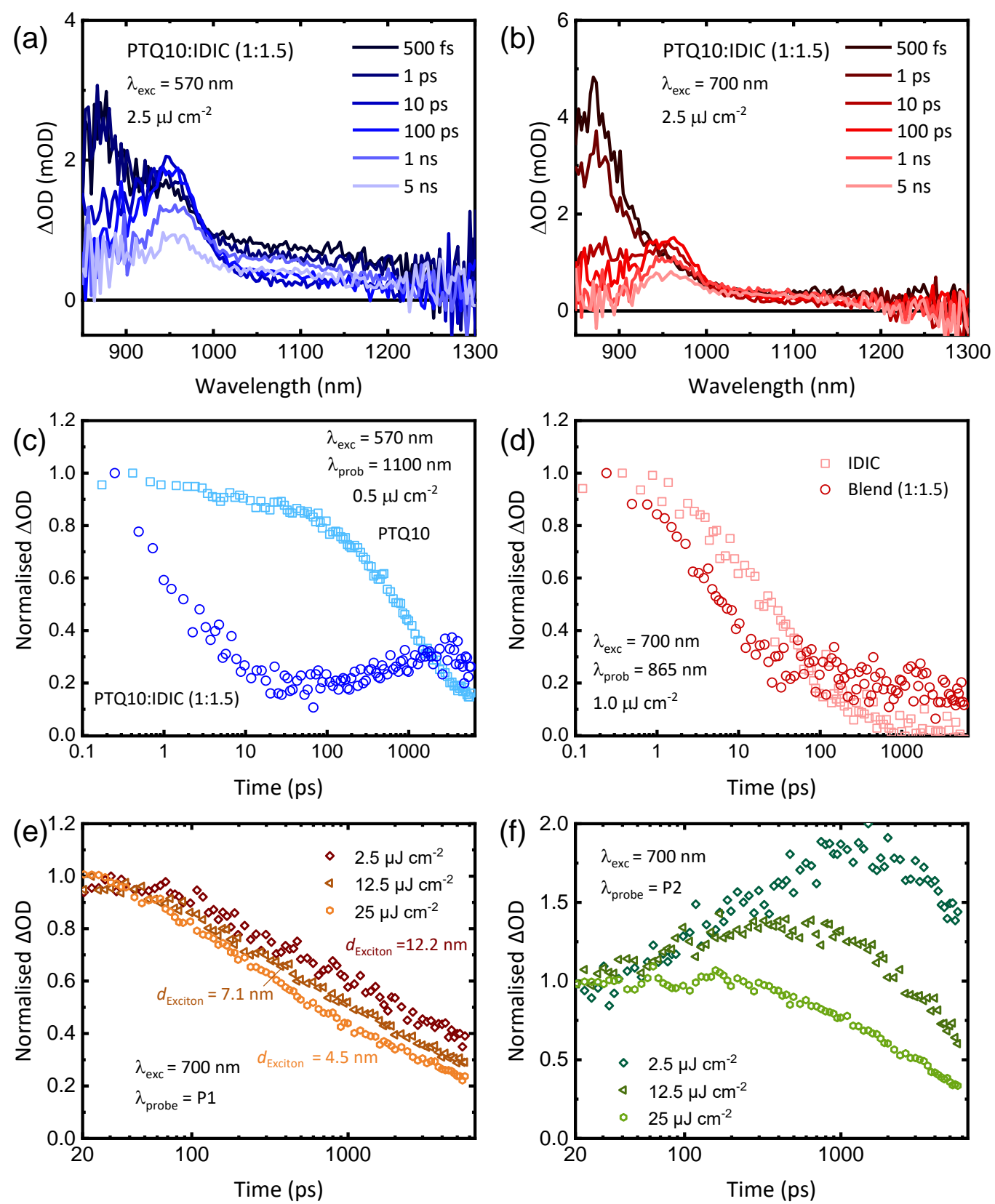

Figure 4. Transient absorption spectra for PTQ10:IDIC (1:1.5) blend film, (a) excited at $570 \mathrm{~nm}$ and (b) excited at $700 \mathrm{~nm}$, with energy density of $2.5 \mu \mathrm{J} \mathrm{cm}^{-2}$. Transient absorption decay dynamics for neat films and PTQ10:IDIC (1:1.5) blend film (c) excited at $570 \mathrm{~nm}$, and (d) excited at $700 \mathrm{~nm}$. Energy density dependent dynamics for PTQ10:IDIC (1:1.5) blend film probed at (e) CT states (P1) including average exciton separations at the initial exciton densities between $5.5 \times 10^{17}$ and $1 \times 10^{19} \mathrm{~cm}^{-3}(2.5-$ $25 \mu \mathrm{J} \mathrm{cm}^{-2}$ ), and (f) CS states (P2) after global analysis.

Transient absorption spectra and corresponding dynamics for the optimised blend film (1:1.5) are shown in Figure 4. Figure 4a presents ultrafast transient absorption spectra after 


\section{WILEY-VCH}

preferentially photoexciting PTQ10 in the PTQ10:IDIC blend (1:1.5) film at $570 \mathrm{~nm}$, assigned to the generation of PTQ10 exciton and subsequent charge generation through an electron transfer process. We note that $570 \mathrm{~nm}$ excitation will excite IDIC as well as PTQ10 excitons, consistent with the early time spectra shown in Figure 4a (we note that ultrafast energy transfer from PTQ10 to IDIC, followed by hole transfer, may also contribute to the data observed at this excitation wavelength). In Figure 4b, analogous spectra for hole transfer are presented after selectively photoexciting IDIC at $700 \mathrm{~nm}$ in the same blend. In these plots, the early time spectra (up to $1 \mathrm{ps)}$ ) are similar to the corresponding IDIC neat film exciton spectra (for 570 and 700 nm excitation, respectively). For both excitation wavelengths, these exciton spectra rapidly evolve (within $10 \mathrm{ps}$ ) to a narrower, red-shifted, PIA peaking at $955 \mathrm{~nm}$ (P1), with this evolution assigned to exciton separation dynamics. At longer times (100 ps - $5 \mathrm{ns)}$ this $955 \mathrm{~nm}$ absorption feature decays, leaving a broader residual absorption (P2) which extends up to $1200 \mathrm{~nm}$. As we discuss further below, we assign the $955 \mathrm{~nm}$ absorption feature (P1) to interfacial CT states, and the broader residual absorption (P2) to separated polarons. ${ }^{[36,68]}$ Figure $4 \mathrm{c}$ shows the PTQ10 exciton decay dynamics at $1005 \mathrm{~nm}$ in the optimised PTQ10:IDIC (1:1.5) blend compared to neat PTQ10 film. It is apparent that in the blend, the PTQ10 exciton decay is significantly faster than that of neat polymer ( 1 ps versus $820 \mathrm{ps})$. These kinetics are in agreement with the PLQ yields of PTQ10 presented above, and indicative of near unity PTQ10 exciton separation efficiency in this blend. The PTQ10 to IDIC Förster energy transfer distance, $R_{0}$, at which the energy transfer efficiency is $50 \%$, is calculated to be $5.9 \mathrm{~nm}$, which is a relatively long due to the strong overlap of donor emission and acceptor absorption and high IDIC absorption coefficient in Figure S1b (See supporting information for calculation details). This suggests that the fast PTQ10 exciton decay may result, at least in part from energy transfer to IDIC).

Analogous data for selective IDIC acceptor excitation at $700 \mathrm{~nm}$ are plotted in Figure 4d. From this data it is apparent that the charge generation kinetics from IDIC excitons $(\sim 3 \mathrm{ps})$ are significantly slower than charge generation kinetics from PTQ10 excitons in the blend film, 


\section{WILEY-VCH}

but faster than the decay to the ground state of IDIC excitons (20 ps) in neat IDIC film. We observed similar texciton decay lifetimes (20 ps) in both the visible (GSB / stimulated emission at $725 \mathrm{~nm}$ ) and in the near-infrared (PIA at $775 \mathrm{~nm}$ ) in Figure S5. These data therefore indicate a sub-unity yield of IDIC exciton separation, in agreement with the relatively lower IDIC PLQ yields reported above (e.g.: 87 and $84 \%$ PL quenching of 1:3 and 1:5 ratios, respectively). Due to high crystallinity of this blend, it is likely that in both cases the kinetics of charge generation transfer are substantially limited by the kinetics of exciton diffusion to donor / acceptor domain interfaces.

Here we consider the excitation fluence dependence of these dynamics, focusing for simplicity on data following selective IDIC excitation, for time delays $>20$ ps to avoid exciton contributions to the data. Global analyses were employed to track the kinetics of the two charge species identified above as a function of excitation pulse energy, as shown in Figures 4e,f, with these kinetics determined from global analysis (see supporting information and Figure S6 for details). The intermediate state (P1, Figure 4e) showed less fluence dependent behaviour, its decay accelerating slightly at higher fluences (see also Figure S7). Analogous kinetics were observed in the visible spectral region (Figure S5), although their assignment is more challenging due to greater spectral overlap. This relatively modest intensity dependence is consistent with the assignment of this feature to bound CT states, with the modest decrease in lifetime with higher intensity (particularly apparent at $100 \mu \mathrm{J} \mathrm{cm}^{-2}$ Figure S7), indicative of increasing contributions from bimolecular recombination of these CT states (we note that under such high excitation densities, the spatial separation of photogenerated states becomes of the order of a few nanometres, equivalent to the spatial delocalisation of bound CT states). ${ }^{[6]}$ The P2 component (Figure 4f) showed a much stronger fluence dependence with increasing excitation density, indicative of its assignment to dissociated polarons. This signal exhibits both $<20$ ps and a slower 100 ps - 1 ns growing phases (Figure $4 \mathrm{f}$ and S7b), with the later coincident with the decay of P1. As such, the initial $(<20 \mathrm{ps})$ rise of P2 is assigned to direct free charge 


\section{WILEY-VCH}

generation from exciton dissociation, and the slower $100 \mathrm{ps}-1 \mathrm{~ns}$ rise to charge generation from interfacial CT states (P1).

As we and others have discussed elsewhere ${ }^{[6,8,20,29,30]}$, bound CT states are primarily formed within mixed domains, with CT states formed at domain boundaries typically readily dissociating into free carriers. As such the P1 species assigned to CT states are likely in relatively amorphous, mixed domains, whilst the P2 species assigned to free charges to crystalline, pure domains, most likely the reason for the striking difference in their photoinduced absorption in the near-infrared. It is hard to rule out some contribution to the data from triplet excitons or triplet CT states at long time delays, although the fluence dependence data in Figure $4 \mathrm{f}$ is indicative of a bimolecular process with a rate constant typical of nongeminate charge recombination. ${ }^{[69-72]}$
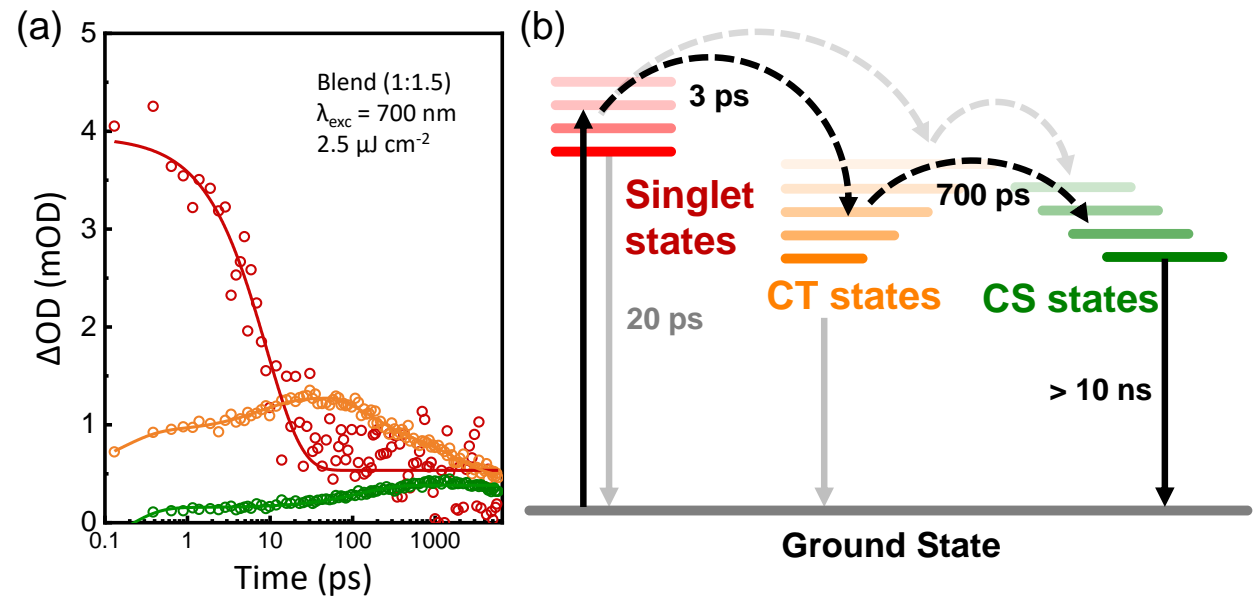

Figure 5. (a) Transient absorption spectral component and relevant kinetics extracted from transient absorption spectra with excitation at $700 \mathrm{~nm}$ for PTQ10:IDIC blend films of 1:1.5 ratio, using the global analysis. (IDIC excitons (red dots), weakly bound charge transfer (CT) states (orange dots) and charge separation (CS) state (Green dots)) (b) Proposed model for charge transfer and separation in blend films of $1: 1.5$ ratio.

Figure 5 summarises the results of global analyses of the transient absorption data for the 1:1.5 blend composition, plotting the populations of IDIC excitons, CT states (P1) and dissociated polarons (P2) as a function of time after $700 \mathrm{~nm}, 2.5 \mu \mathrm{J} \mathrm{cm} \mathrm{cm}^{-2}$. This optimised 


\section{WILEY-VCH}

PTQ10:IDIC blend shows an IDIC singlet exciton decay time of circa 3 ps (red circles), aligned with a simultaneous rise of interfacial CT states (orange dots). This exciton separation is in kinetic competition with exciton decay to ground (20 ps in neat IDIC films). Subsequently interfacial CT states dissociate to free charges with a half-lifetime of 700 ps. These kinetics are summarised in Figure 5b. We also note the presence of some CT and free charge generation at early times, also indicated by the grey dashed arrows in Figure 5b. The decay time of P2 spectral feature were observed to be strongly intensity dependent (Figure S8), consistent with our assignment of this feature to dissociated polarons. As shown in Figure S9, in the 1:0.5 blend, CT state formation is enhanced, most likely associated with the greater molecular intermixing in this blend. However, in 1:5 blends, lower CT state formation is observed, consistent with the greater dominance of interfaces between PTQ10 and pure IDIC domains.

\section{Discussion}

This study focuses on providing some insight into the correlation between blend morphology / crystallinity and charge separation dynamics in devices based on PTQ10:IDIC blends. GIWAXS analysis indicates this blend composition exhibits remarkably high crystallinity, with molecular stacking coherence lengths exceeding those reported previously for polymer:NFA blends ( 8.5 and $10.7 \mathrm{~nm}$ for PTQ10 and IDIC respectively). Analyses of the photophysics for neat materials yielded also remarkably long exciton diffusion lengths for both PTQ10 (19 nm) and IDIC (9.5 nm). For PTQ10, its long exciton diffusion length is assigned primarily to its long exciton lifetime (i.e.: from suppressed non-radiative decay to ground), whilst for IDIC it is assigned to a high exciton diffusion coefficient consistent with its strong $\pi-\pi$ stacking, partly offset by a rather short exciton lifetime. Given the high crystallinity, and large domain sizes, indicated by the GIWAXS data, these long exciton diffusion lengths of PTQ10 ( 820 ps) are

critical to enabling efficient exciton dissociation in these films, as confirmed by photoluminescence quenching data. Therefore, electron transfer from PTQ10 to IDIC is highly 


\section{WILEY-VCH}

efficient, showing over 99\% PL quenching, which is consistent with fast exciton decay of PTQ10 in blends compared to neat PTQ10 ( 1 ps versus 820 ps). Hole transfer from IDIC to PTQ10 is relatively less efficient particularly for higher IDIC content blends (87 and 84\% PL quenching for 1:3 and 1:5 blends respectively). In the optimised 1:1.5 blend, slow IDIC exciton separation ( $\sim 3$ ps relative to the exciton lifetime of $20 \mathrm{ps}$ in neat IDIC films) results in relatively inefficient charge generation. This is attributed to exciton diffusion limitations) due to the presence of large pure domains of IDIC, particularly at high IDIC blend compositions as shown in GIWAXS data. In blends, we observed two intermediate states after exciton dissociation; interfacial bound CT states and separated polaron states. Of particular note, we observed CT state decay coincides with a rise in separated polarons, indicating a significant fraction of interfacial CT states separate rather than undergoing geminate recombination to ground. Charge collection at short circuit is observed to be highly efficient up to 2 suns, attributed to a high blend effective mobility (resulting from the blends high crystallinity and face on molecular orientation) and most likely due to suppressed bimolecular recombination by strong phase segregation in Figure S10. We conclude the main factors determining photocurrent generation are exciton and CT state separation, rather than charge collection, consistent with our observation that $J_{\mathrm{SC}}$ increases linearly with light intensity.

The optimised 1:1.5 PTQ10:IDIC blend exhibits EQEs up to 80\%. From the analyses herein, this EQE is not limited by bimolecular recombination losses. Rather this EQE is likely limited by reflection / interference losses and also by some CT state recombination within relatively increased mixed phases. We note that quantifying the magnitude of CT recombination losses limiting $J_{\mathrm{SC}}$ in complete devices would require further studies, such as time delayed charge collection, beyond the scope of this study. Blends with higher IDIC compositions show lower EQEs, most likely due to less efficient exciton dissociation resulting from IDIC domain sizes exceeding its exciton diffusion length. The 1:0.5 blend, with a lower IDIC composition, shows lower EQEs and also a loss of fill factor, most likely resulting from enhanced (field 


\section{WILEY-VCH}

dependent) CT state recombination due to a greater proportion of molecularly intermixed phase. This enhanced CT state recombination is consistent with the observation of CT state PL and EL for this blend. (Figure S2).

It is striking that our data indicates free charge generation in PTQ10:IDIC blends substantially results from CT state separation with a time constant of $\sim 700 \mathrm{ps}$. In several other NFA blends, CT state formation has been observed to result in (field dependent) CT state recombination losses, limiting device EQE and FF. CT state formation and recombination losses in blends with both fullerene and non-fullerene acceptors have been shown to be significantly determined by donor / acceptor energetics and by blend morphology, with smaller HOMO or LUMO energy offsets and more intermixed blend morphologies increasing CT state recombination losses. ${ }^{[9,20,28,32,73-75]}$ However, surprisingly the PTQ10:IDIC blend device with a small HOMO energy offset $(200 \mathrm{meV})$ exhibited efficient CT state dissociation rather than significant $\mathrm{CT}$ state recombination to the ground state, which is consistent with a recent study showing efficient charge generation from barrierless CT state dissociation. ${ }^{[39]}$

It appears most likely that the ability of CT states formed in PTQ10:IDIC blends to undergo efficient separation to free carriers results from the high crystallinity of this blend. The presence of highly crystalline domains with strong $\pi-\pi$ stacking, face-on orientation parallel to the substrate and high carrier mobility appears to assist spatial charge separation and promotes free charge generation and collection. We note that we have previously estimated a CT state radius of 3-5 nm in PCDTBT:PCBM blends. ${ }^{[6]}$ The modest intensity dependence of P1's decay kinetics in Figure 4e is indicative of a similar CT radius for PTQ10:IDIC. As such, given the high crystallinity of PTQ10:IDIC, it is likely that most bound CT states formed in molecularly mixed domains will be able to access crystalline, pure domains, consistent with our observation of efficient CT state dissociation into free charges.

Here we have investigated the impact of film morphology on photocurrent generation and charge carrier recombination losses in high crystalline NFA-based OSCs. The high 


\section{WILEY-VCH}

crystallinity of PTQ10:IDIC optimised blends enables the efficient exciton diffusion and charge transport but also promotes the CT state dissociation to free charge generation. This efficient charge generation process contributes to an enhanced photocurrent generation in the device despite a relatively small HOMO energy offset. Our results emphasises the potential for high material crystallinity to enhance charge separation and collection in OSCs, but also that, due to the formation of crystalline domains, long exciton diffusion lengths are likely to be prerequisite for efficient exciton separation in such high crystallinity devices. 


\section{WILEY-VCH}

\section{Supporting Information}

Supporting Information is available from the Wiley Online Library or from the author.

\section{Acknowledgements}

The authors gratefully acknowledge funding from supported by KAUST under the Grant Agreement number OSR-2015-CRG4-2572 and the EPSRC/GCRF project SUNRISE (EP/P032591/1). H. C. acknowledges Basic Science Research Program through the National Research Foundation of Korea (NRF) funded by the Ministry of Education (2018R1A6A3A03011245). J.Z. acknowledges a PhD scholarship from China Scholarship Council (201503170255).

Received: ((will be filled in by the editorial staff))

Revised: ((will be filled in by the editorial staff)) Published online: ((will be filled in by the editorial staff))

\section{References}

[1] H. Cha, J. Wu, A. Wadsworth, J. Nagitta, S. Limbu, S. Pont, Z. Li, J. Searle, M. F. Wyatt, D. Baran, J. S. Kim, I. McCulloch, J. R. Durrant, Adv. Mater. 2017, 29, 1701156.

[2] C. Sun, F. Pan, H. Bin, J. Zhang, L. Xue, B. Qiu, Z. Wei, Z. G. Zhang, Y. Li, Nat. Commun. 2018, 9, 743.

[3] Q. Fan, Y. Wang, M. Zhang, B. Wu, X. Guo, Y. Jiang, W. Li, B. Guo, C. Ye, W. Su, J. Fang, X. Ou, F. Liu, Z. Wei, T. C. Sum, T. P. Russell, Y. Li, Adv. Mater. 2018, 30, 1704546.

[4] L. Meng, Y. Zhang, X. Wan, C. Li, X. Zhang, Y. Wang, X. Ke, Z. Xiao, L. Ding, R. Xia, H. L. Yip, Y. Cao, Y. Chen, Science. 2018, 361, 1094.

[5] W. Ma, J. R. Tumbleston, M. Wang, E. Gann, F. Huang, H. Ade, Adv. Energy Mater. 2013, 3, 864 .

[6] H. Utzat, S. D. Dimitrov, S. Wheeler, E. Collado-Fregoso, P. S. Tuladhar, B. C. Schroeder, I. McCulloch, J. R. Durrant, J. Phys. Chem. C 2017, 121, 9790.

[7] C. Kästner, K. Vandewal, D. A. M. Egbe, H. Hoppe, Adv. Sci. 2017, 4, 9.

[8] M. Scarongella, A. A. Paraecattil, E. Buchaca-Domingo, J. D. Douglas, S. Beaupré, T. McCarthy-Ward, M. Heeney, J. E. Moser, M. Leclerc, J. M. J. Fréchet, N. Stingelin, N. Banerji, J. Mater. Chem. A 2014, 2, 6218. 


\section{WILEY-VCH}

[9] S. Sweetnam, K. R. Graham, G. O. Ngongang Ndjawa, T. Heumüller, J. A. Bartelt, T. M. Burke, W. Li, W. You, A. Amassian, M. D. McGehee, J. Am. Chem. Soc. 2014, $136,14078$.

[10] C. Cui, Y. Li, Energy Environ. Sci. 2019, 12, 3225-3246.

[11] A. Wadsworth, H. Bristow, Z. Hamid, M. Babics, N. Gasparini, C. W. Boyle, W. Zhang, Y. Dong, K. J. Thorley, M. Neophytou, R. S. Ashraf, J. R. Durrant, D. Baran, I. McCulloch, Adv. Funct. Mater. 2019, 29, 1808429.

[12] C. Kästner, K. Vandewal, D. A. M. Egbe, H. Hoppe, Adv. Sci. 2017, 4, 1600331.

[13] G. Sini, M. Schubert, C. Risko, S. Roland, O. P. Lee, Z. Chen, T. V. Richter, D. Dolfen, V. Coropceanu, S. Ludwigs, U. Scherf, A. Facchetti, J. M. J. Fréchet, D. Neher, Adv. Energy Mater. 2018, 8, 1702232.

[14] M. Azzouzi, J. Yan, T. Kirchartz, K. Liu, J. Wang, H. Wu, J. Nelson, Phys. Rev. X 2018, 8, 31055.

[15] A. Gluchowski, K. L. G. Gray, S. N. Hood, I. Kassal, J. Phys. Chem. Lett. 2018, 9, 1359.

[16] J. Kurpiers, T. Ferron, S. Roland, M. Jakoby, T. Thiede, F. Jaiser, S. Albrecht, S. Janietz, B. A. Collins, I. A. Howard, D. Neher, Nat. Commun. 2018, 9, 2038.

[17] Z. Zheng, N. R. Tummala, Y. T. Fu, V. Coropceanu, J. L. Brédas, ACS Appl. Mater. Inter. 2017, 9, 18095.

[18] S. Sweetnam, R. Prasanna, T. M. Burke, J. A. Bartelt, M. D. McGehee, J. Phys. Chem. C 2016, 120, 6427.

[19] S.-U.-Z. Khan, G. Londi, X. Liu, M. A. Fusella, G. D’Avino, L. Muccioli, A. N. Brigeman, B. Niesen, T. C.-J. Yang, Y. Olivier, J. T. Dull, N. C. Giebink, D. Beljonne, B. P. Rand, Chem. Mater. 2019, 31, 6808.

[20] S. D. Dimitrov, M. Azzouzi, J. Wu, J. Yao, Y. Dong, P. S. Tuladhar, B. C. Schroeder, E. R. Bittner, I. McCulloch, J. Nelson, J. R. Durrant, J. Am. Chem. Soc. 2019, 141, 4634.

[21] F. C. Jamieson, E. B. Domingo, T. McCarthy-Ward, M. Heeney, N. Stingelin, J. R. Durrant, Chem. Sci. 2012, 3, 485.

[22] S. Shoaee, S. Subramaniyan, H. Xin, C. Keiderling, P. S. Tuladhar, F. Jamieson, S. A. Jenekhe, J. R. Durrant, Adv. Funct. Mater. 2013, 23, 3286.

[23] A. A. Bakulin, A. Rao, V. G. Pavelyev, P. H. M. Van Loosdrecht, M. S. Pshenichnikov, D. Niedzialek, J. Cornil, D. Beljonne, R. H. Friend, Science. 2012, 335, 1340. 


\section{WILEY-VCH}

[24] Y. Tamai, Y. Fan, V. O. Kim, K. Ziabrev, A. Rao, S. Barlow, S. R. Marder, R. H. Friend, S. M. Menke, ACS Nano 2017, 11, 12473.

[25] N. D. Eastham, A. S. Dudnik, T. J. Aldrich, E. F. Manley, T. J. Fauvell, P. E. Hartnett, M. R. Wasielewski, L. X. Chen, F. S. Melkonyan, A. Facchetti, R. P. H. Chang, T. J. Marks, Chem. Mater. 2017, 29, 4432.

[26] Y. W. Soon, S. Shoaee, R. S. Ashraf, H. Bronstein, B. C. Schroeder, W. Zhang, Z. Fei, M. Heeney, I. McCulloch, J. R. Durrant, Adv. Funct. Mater. 2014, 24, 1474.

[27] H. Hu, P. C. Y. Chow, G. Zhang, T. Ma, J. Liu, G. Yang, H. Yan, Acc. Chem. Res. 2017, 50, 2519.

[28] H. Cha, G. Fish, J. Luke, A. Alraddadi, H. H. Lee, W. Zhang, Y. Dong, S. Limbu, A. Wadsworth, I. P. Maria, L. Francàs, H. L. Sou, T. Du, J. S. Kim, M. A. McLachlan, I. McCulloch, J. R. Durrant, Adv. Energy Mater. 2019, 9, 1901254.

[29] M. Causa, J. De Jonghe-Risse, M. Scarongella, J. C. Brauer, E. Buchaca-Domingo, J. E. Moser, N. Stingelin, N. Banerji, Nat. Commun. 2016, 7, 12556.

[30] M. Scarongella, J. De Jonghe-Risse, E. Buchaca-Domingo, M. Causa, Z. Fei, M. Heeney, J. E. Moser, N. Stingelin, N. Banerji, J. Am. Chem. Soc. 2015, 137, 2908.

[31] J. Razzell-Hollis, S. Limbu, J. S. Kim, J. Phys. Chem. C 2016, 120, 10806.

[32] A. C. Jakowetz, M. L. Böhm, J. Zhang, A. Sadhanala, S. Huettner, A. A. Bakulin, A. Rao, R. H. Friend, J. Am. Chem. Soc. 2016, 138, 11672.

[33] E. Collado-Fregoso, F. Deledalle, H. Utzat, P. S. Tuladhar, S. D. Dimitrov, A. Gillett, C. H. Tan, W. Zhang, I. McCulloch, J. R. Durrant, Adv. Funct. Mater. 2017, 27, 1604426.

[34] S. D. Dimitrov, J. R. Durrant, Chem. Mater. 2014, 26, 616.

[35] J. Zhang, Q. Gu, T. T. Do, K. Rundel, P. Sonar, R. H. Friend, C. R. McNeill, A. A. Bakulin, J. Phys. Chem. A 2018, 122, 1253.

[36] B. Kan, J. Zhang, F. Liu, X. Wan, C. Li, X. Ke, Y. Wang, H. Feng, Y. Zhang, G. Long, R. H. Friend, A. A. Bakulin, Y. Chen, Adv. Mater. 2018, 30, 1704904.

[37] D. Baran, N. Gasparini, A. Wadsworth, C. H. Tan, N. Wehbe, X. Song, Z. Hamid, W. Zhang, M. Neophytou, T. Kirchartz, C. J. Brabec, J. R. Durrant, I. McCulloch, Nat. Commun. 2018, 9, 2059.

[38] H. Cha, C. H. Tan, J. Wu, Y. Dong, W. Zhang, H. Chen, S. Rajaram, K. S. Narayan, I. McCulloch, J. R. Durrant, Adv. Energy Mater. 2018, 8, 1801537. 


\section{WILEY-VCH}

[39] L. Perdigón-Toro, H. Zhang, A. Markina, J. Yuan, S. M. Hosseini, C. M. Wolff, G. Zuo, M. Stolterfoht, Y. Zou, F. Gao, D. Andrienko, S. Shoaee, D. Neher, Adv. Mater. 2020, 32, 1906763.

[40] Y. Zhong, M. Causa', G. J. Moore, P. Krauspe, B. Xiao, F. Günther, J. Kublitski, R. Shivhare, J. Benduhn, E. BarOr, S. Mukherjee, K. M. Yallum, J. Réhault, S. C. B. Mannsfeld, D. Neher, L. J. Richter, D. M. DeLongchamp, F. Ortmann, K. Vandewal, E. Zhou, N. Banerji, Nat. Commun. 2020, 11, 388.

[41] J. Yuan, Y. Zhang, L. Zhou, G. Zhang, H.-L. Yip, T.-K. Lau, X. Lu, C. Zhu, H. Peng, P. A. Johnson, M. Leclerc, Y. Cao, J. Ulanski, Y. Li, Y. Zou, Joule 2019, 3, 1140.

[42] S. Li, L. Zhan, C. Sun, H. Zhu, G. Zhou, W. Yang, M. Shi, C. Z. Li, J. Hou, Y. Li, H. Chen, J. Am. Chem. Soc. 2019, 141, 3073.

[43] Y. Lin, F. Zhao, Y. Wu, K. Chen, Y. Xia, G. Li, S. K. K. Prasad, J. Zhu, L. Huo, H. Bin, Z. G. Zhang, X. Guo, M. Zhang, Y. Sun, F. Gao, Z. Wei, W. Ma, C. Wang, J. Hodgkiss, Z. Bo, O. Inganäs, Y. Li, X. Zhan, Adv. Mater. 2017, 29, 1604155.

[44] H. Cha, S. Wheeler, S. Holliday, S. D. Dimitrov, A. Wadsworth, H. H. Lee, D. Baran, I. McCulloch, J. R. Durrant, Adv. Funct. Mater. 2018, 28, 1704389.

[45] N. A. Ran, S. Roland, J. A. Love, V. Savikhin, C. J. Takacs, Y. T. Fu, H. Li, V. Coropceanu, X. Liu, J. L. Brédas, G. C. Bazan, M. F. Toney, Di. Neher, T. Q. Nguyen, Nat. Commun. 2017, 8, 79.

[46] H. Hu, K. Jiang, P. C. Y. Chow, L. Ye, G. Zhang, Z. Li, J. H. Carpenter, H. Ade, H. Yan, Adv. Energy Mater. 2018, 8, 1701674.

[47] K. Zhou, Y. Liu, A. Alotaibi, J. Yuan, C. Jiang, J. Xin, X. Liu, B. A. Collins, F. Zhang, W. Ma, ACS Energy Lett. 2020, 5, 589.

[48] C. Sun, S. Qin, R. Wang, S. Chen, F. Pan, B. Qiu, Z. Shang, L. Meng, C. Zhang, M. Xiao, C. Yang, Y. Li, J. Am. Chem. Soc. 2020, 3, 1465.

[49] Z. Fei, F. D. Eisner, X. Jiao, M. Azzouzi, J. A. Röhr, Y. Han, M. Shahid, A. S. R. Chesman, C. D. Easton, C. R. McNeill, T. D. Anthopoulos, J. Nelson, M. Heeney, Adv. Mater. 2018, 30, 1705209.

[50] M. A. Faist, T. Kirchartz, W. Gong, R. S. Ashraf, I. McCulloch, J. C. De Mello, N. J. Ekins-Daukes, D. D. C. Bradley, J. Nelson, J. Am. Chem. Soc. 2012, 134, 685.

[51] N. Gasparini, A. Salleo, I. McCulloch, D. Baran, Nat. Rev. Mater. 2019, 4, 229.

[52] L. Ke, J. Min, M. Adam, N. Gasparini, Y. Hou, J. D. Perea, W. Chen, H. Zhang, S. Fladischer, A. C. Sale, E. Spiecker, R. R. Tykwinski, C. J. Brabec, T. Ameri, $A d v$. Energy Mater. 2016, 6, 1502355. 


\section{WILEY-VCH}

[53] L. Zhan, S. Li, T. Lau, Y. Cui, X. Lu, M. Shi, C. Li, H. Li, J. Hou, H. Chen, Energy Environ. Sci. 2020, 13, 635-645 .

[54] J. Wu, J. Luke, H. K. H. Lee, P. S. Tuladhar, H. Cha, Y. Jang, W. C. Tsoi, M. Heeney, H. Kang, K. Lee, T. Kirchartz, J. Kim, J. R. Durrant, Nat. Commun. 2019, 10, 5159.

[55] L. Zhang, B. Lin, Z. Ke, J. Chen, W. Li, M. Zhang, W. Ma, Nano Energy 2017, 41, 609.

[56] X. Li, F. Pan, C. Sun, M. Zhang, Z. Wang, J. Du, J. Wang, M. Xiao, L. Xue, Z. G. Zhang, C. Zhang, F. Liu, Y. Li, Nat. Commun. 2019, 10, 519.

[57] Y. Lin, Q. He, F. Zhao, L. Huo, J. Mai, X. Lu, C. J. Su, T. Li, J. Wang, J. Zhu, Y. Sun, C. Wang, X. Zhan, J. Am. Chem. Soc. 2016, 138, 2973.

[58] H. K. H. Lee, J. Wu, J. Barbé, S. M. Jain, S. Wood, E. M. Speller, Z. Li, F. A. Castro, J. R. Durrant, W. C. Tsoi, J. Mater. Chem. A 2018, 6, 5618.

[59] H. Bristow, K. J. Thorley, A. J. P. White, A. Wadsworth, M. Babics, Z. Hamid, W. Zhang, A. F. Paterson, J. Kosco, J. Panidi, T. D. Anthopoulos, I. McCulloch, Adv. Electron. Mater. 2019, 5, 1900344.

[60] D. Baran, T. Kirchartz, S. Wheeler, S. Dimitrov, M. Abdelsamie, J. Gorman, R. S. Ashraf, S. Holliday, A. Wadsworth, N. Gasparini, P. Kaienburg, H. Yan, A. Amassian, C. J. Brabec, J. R. Durrant, I. McCulloch, Energy Environ. Sci. 2016, 9, 3783.

[61] C. Sun, F. Pan, S. Chen, R. Wang, R. Sun, Z. Shang, B. Qiu, J. Min, M. Lv, L. Meng, C. Zhang, M. Xiao, C. Yang, 2019, 31, 1905480.

[62] Y. Lin, F. Zhao, Q. He, L. Huo, Y. Wu, T. C. Parker, W. Ma, Y. Sun, C. Wang, D. Zhu, A. J. Heeger, S. R. Marder, X. Zhan, J. Am. Chem. Soc. 2016, 138, 4955.

[63] L. Ye, W. Zhao, S. Li, S. Mukherjee, J. H. Carpenter, O. Awartani, X. Jiao, J. Hou, H. Ade, Adv. Energy Mater. 2017, 7, 1602000.

[64] J. K. Gallaher, S. K. K. Prasad, M. A. Uddin, T. Kim, J. Y. Kim, H. Y. Woo, J. M. Hodgkiss, Energy Environ. Sci. 2015, 8, 2713.

[65] S. Chandrabose, K. Chen, A. J. Barker, J. J. Sutton, S. K. K. Prasad, J. Zhu, J. Zhou, K. C. Gordon, Z. Xie, X. Zhan, J. M. Hodgkiss, J. Am. Chem. Soc. 2019, 141, 6922.

[66] S. Dai, S. Chandrabose, J. Xin, T. Li, K. Chen, P. Xue, K. Liu, K. Zhou, W. Ma, J. M. Hodgkiss, X. Zhan, J. Mater. Chem. A 2019, 7, 2268.

[67] S. D. Dimitrov, B. C. Schroeder, C. B. Nielsen, H. Bronstein, Z. Fei, I. McCulloch, M. Heeney, J. R. Durrant, Polymers. 2016, 8, 14.

[68] O. M. Awartani, B. Gautam, W. Zhao, R. Younts, J. Hou, K. Gundogdu, H. Ade, J. Mater. Chem. A 2018, 6, 12484. 


\section{WILEY-VCH}

[69] A. Rao, P. C. Y. Chow, S. Gélinas, C. W. Schlenker, C. Z. Li, H. L. Yip, A. K. Y. Jen, D. S. Ginger, R. H. Friend, Nature 2013, 500, 435.

[70] P. C. Y. Chow, S. Gélinas, A. Rao, R. H. Friend, J. Am. Chem. Soc. 2014, 9, 3424.

[71] X. K. Chen, T. Wang, J. L. Brédas, Adv. Energy Mater. 2017, 7, 1602713.

[72] Q. Sun, C. Ni, Y. Yu, S. Attique, S. Wei, Z. Ci, J. Wang, S. Yang, J. Mater. Chem. C 2018, 6, 12484.

[73] S. M. Menke, A. Cheminal, P. Conaghan, N. A. Ran, N. C. Greehnam, G. C. Bazan, T. Q. Nguyen, A. Rao, R. H. Friend, Nat. Commun. 2018, 9, 277.

[74] S. D. Dimitrov, A. A. Bakulin, C. B. Nielsen, B. C. Schroeder, J. Du, H. Bronstein, I. McCulloch, R. H. Friend, J. R. Durrant, J. Am. Chem. Soc. 2012, 134, 18189.

[75] T. M. Clarke, A. M. Ballantyne, J. Nelson, D. D. C. Bradley, J. R. Durrant, Adv. Funct. Mater. 2008, 18, 4029. 


\section{WILEY-VCH}

The ability of high materials crystallinity to enhance charge separation and collection in organic solar cells (OSCs) has been demonstrated, but also that long exciton diffusion lengths are essential for efficient exciton separation in such high crystallinity devices. As such it provides new insights into materials and films design requirements for OSCs employing nonfullerene acceptors, and in particular the advantages, and also limitations, of employing highly crystalline blends in such devices.

\section{Keyword}

organic solar cells; nonfullerene acceptor; charge separation; recombination; phase separation

\section{Authors}

H. Cha, Y. Zheng, Y. Dong, H. H. Lee, J. Wu, H. Bristow, J. Zhang, H. K. H. Lee, W. C. Tsoi, A. A. Bakulin, I. McCulloch, J. R. Durrant

\section{Title}

Exciton and Charge Carrier Dynamics in Highly Crystalline PTQ10:IDIC Organic Solar Cells.

\section{ToC figure}

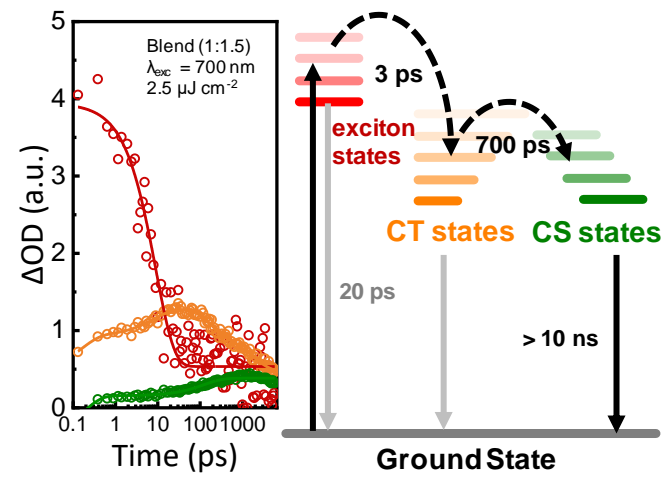

